\title{
MOLECULAR DYNAMICS SIMULATIONS OF THE RICHTMYER- MESHKOV INSTABILITY IN SHOCK LOADED SOLIDS
}

\author{
S. V. Zybin ${ }^{1}$, V. V. Zhakhovskii ${ }^{2}$, E. M. Bringa ${ }^{3}$, S. I. Abarzhi ${ }^{4}$, and B. Remington ${ }^{3}$ \\ ${ }^{1}$ Materials and Process Simulation Center, California Institute of Technology, Pasadena, CA 91125, USA \\ ${ }^{2}$ Institute of Laser Engineering, Osaka University, Osaka, 565-0871, Japan \\ ${ }^{3}$ Lawrence Livermore National Laboratory, Livermore CA 94550, USA \\ ${ }^{4}$ Center for Turbulence Research, Stanford, CA 94305, FLASH Center, The University of Chicago, IL 60637
}

\begin{abstract}
For the first time, molecular dynamics simulations (MD) are applied to study the evolution of shock-driven Richtmyer-Meshkov instability of the perturbations at a solid surface and at the interface separating two solids of different densities. We study the dependence of the instability evolution on the strength and orientation of the shock, and analyze the structure of the material in a vicinity of the interface perturbations (i.e. the spikes and bubbles).
\end{abstract}

Keywords: molecular dynamics, elastic-plastic shock, instability, Richtmyer-Meshkov, shear strength PACS: $02.70 . \mathrm{Ns}, 47.20 . \mathrm{Bp}, 52.57 . \mathrm{Fg}, 62.50 .+\mathrm{p}, 68.35 . \mathrm{Ja}$

\section{INTRODUCTION}

The dynamics of a solid under deformations of very high strain rate and the development of instabilities at the solid interfaces are of great interest in inertial confinement fusion (ICF), detonations in heterogeneous (e.g. polymerbonded) explosive materials, ejecta formation, and many other applications. It is also one of key issues for theories and models, which study material strength and phase transitions under a high strain rates. The instabilities developed include the Rayleigh-Taylor (RTI) and Richtmyer-Meshkov (RMI) instabilities, and they occur at interfaces between two solids subjected to a sustained and impulsive shock-driven acceleration respectively. In solids RTI and RMI develop if the value of the acceleration (initial shock) exceeds a certain threshold, which is set by the material properties and can be used to measure these properties under high strain rate deformation. Such phenomena have been studied both experimentally [1-4] and theoretically [5-7], and recently by the molecular dynamics (MD) approach [8-9]. Here, we report some preliminary results of MD simulations of the evolution of the shock-driven RMI in solids.

\section{SIMULATION APPROACH}

We conducted MD simulations of solids, when a planar shock wave refracts through the perturbed interface between 1) two different solids, and 2) a solid and a vacuum. For the solid-solid interface, we consider a system composed of inert-gas crystals with different densities, light $\left(\rho_{1}\right)$ and heavy $\left(\rho_{2}\right)$. The density ratio is $\rho_{1} / \rho_{2}=1 / 4$. We considered the two cases of the shock propagation: from the light to heavy solid and from the heavy to light solid. The simulations in a system with solidvacuum interface correspond to experiments in which a metal with corrugations at the free surface is shocked from the opposite side.

For solid-solid case, the atomic interaction is modeled via a modified Lennard-Jones (LJ) pair potential with an additional spline term to provide smooth second derivatives [10]. The potential parameters are used to define reduced MD units $(m d u)$. For argon, length $m d u \sigma=3.405 \AA$, energy 


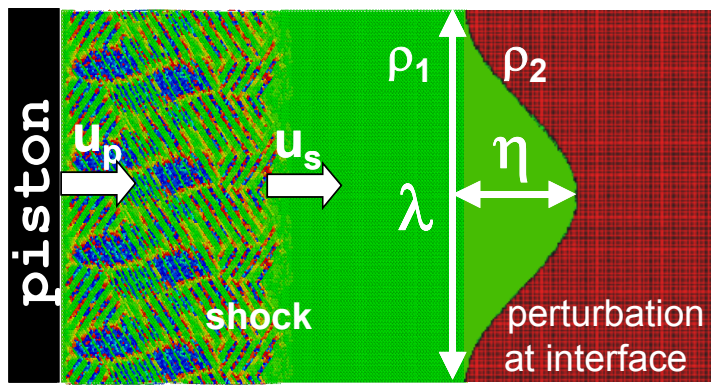

FIGURE 1. Simulation of a piston-driven planar shock propagation through the perturbed interface separating 1) two solids of different densities $\left(\rho_{1}<\rho_{2}\right)$, and 2) solid and vacuum $\left(\rho_{2}=0\right)$.

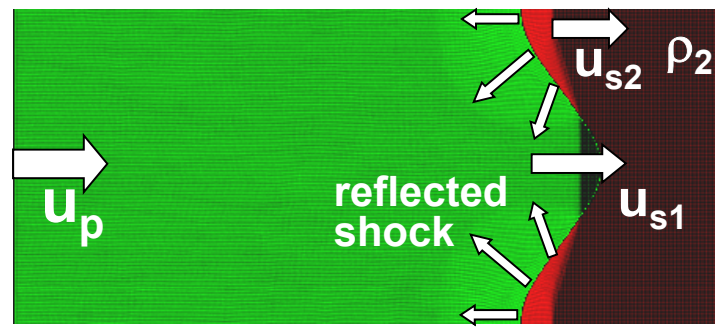

FIGURE 2. Shock wave passes through the light-toheavy solid interface $\left(\rho_{2}=4 \rho_{1}, \eta=\lambda / 3=28 \mathrm{~nm}\right)$ generating a slower second shock in the denser solid and a reflected shock running backward into the light material.

$m d u \quad \varepsilon / \mathrm{k}_{\mathrm{B}}=119.8 \mathrm{~K}$, time $\left.m d u=\sigma\left(\left(\mathrm{m}_{\mathrm{a}} / 48\right) / \varepsilon\right)\right)^{1 / 2}$ $=0.311 \mathrm{ps}$, velocity $m d u=1.094 \mathrm{~km} / \mathrm{s}$, and pressure $m d u$ is $41.9 \mathrm{MPa}$. The system is built from two $\mathrm{LJ}$ solids that differ only by the atomic mass: light $\left(\mathrm{m}_{1}=\mathrm{m}_{\mathrm{a}}\right)$ and heavy $\left(\mathrm{m}_{2}=4 \mathrm{~m}_{\mathrm{a}}\right)$. About 20 million atoms were used to build the solid-solid system with dimensions $108 \times 15 \times 487 \mathrm{~nm}$. For MD simulations of the RM instability of metal-tovacuum surface we chose Mishin's potential for copper [11] which has been previously used to study shocks in single crystal and polycrystalline copper [12-13]. The copper supercell has about 6 million atoms and sizes of $74 \times 5.1 \times 218 \mathrm{~nm}$. Periodic boundaries were imposed in both transverse directions.

Figure 1 shows a characteristic setup for modeling RMI in planar geometry. A piston wall represented by the repulsive potential moves at constant velocity $u_{p}$ and generates a planar shock wave moving at the velocity $u_{s}$ in the first solid. Then the shock waves passes into the second solid (or vacuum) through the interface with a single- wave perturbation of the amplitude $\eta$ and wavelength $\lambda$ set by the largest transverse dimension (roughly, $\lambda \sim 100 \mathrm{~nm}$ ). The ratio $\eta / \lambda$ in most simulations was chosen about $0.25-0.33$.

\section{RESULTS AND DISCUSSION}

In the first series of runs, we have studied the planar shock moving from the light to heavy solid. Both solids have the [110] orientation along the shock direction. Figure 2 shows a snapshot when the first shock wave $u_{s l}$ hits the interface and splits into two non-planar waves: transmitted shock $u_{s 2}$ and reflected shock moving back into the light solid. After reaching the piston this wave reflects back and re-shocks the interface. Remarkably, the interface perturbation is shrinking when the shock passes through it, but then it bounces back and may start to grow. The growth rate strongly depends on the initial shock strength (see Fig. 3). For instance, there is almost no growth at the piston velocity $u_{p}=$ $0.7 \mathrm{mdu}$ (approximately $0.54 \mathrm{c}_{1}$, where $\mathrm{c}_{1}$ is the sound velocity in the light argon solid) until the transmitted shock emerges from an open surface of the heavy solid and the rarefaction wave moves back to the interface (see Fig. 3a). At higher velocity of the piston $u_{p}=1.0 \mathrm{mdu}$, the growth starts as soon as the shock passes through the interface, and then, when the rarefaction wave from the open right side reaches the interface, its rate increases. Such threshold behavior in solids is essentially different from that for RMI in liquids and indicates strong dependence of the growth conditions on the material properties (e.g. shear strength), generation of dislocations, defects, and plastic flow. There is a possibility of local melting in vicinity of the interface even though a chosen piston velocity does not reach the critical value at which the light solid could start to melt. This issue is currently under investigation and will be reported in a subsequent paper.

In the second series of runs, we have studied the evolution of the interface for a shock wave moving from heavy to light solid. In this case, instead of reflected shock, a non-planar rarefaction wave moves backward into the first (heavy) material as shown in Fig. 4. The transmitted shock wave $u_{s l}$ also becomes non-planar, and the front oscillates, but the front fluctuations decay soon. The 


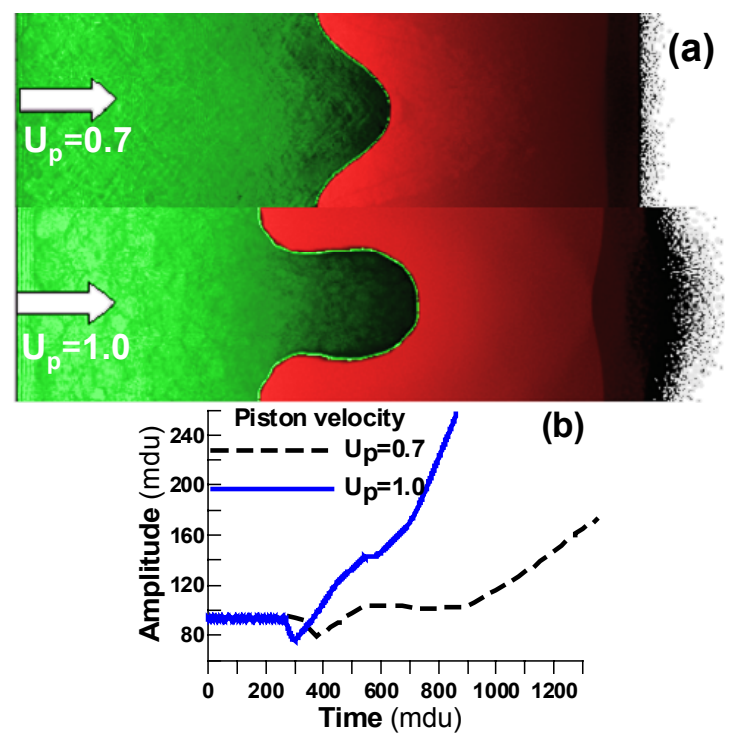

FIGURE 3. The results for piston velocities 0.7 and 1.0 $m d u, \eta=\lambda / 3=36 \mathrm{~nm}$ : (a) snapshots of the perturbation at the final stage (when passed [110] shock wave emerges from a surface of the second solid and the rarefaction wave runs back to the interface); (b) evolution of the perturbation amplitude.

transmitted shock continues to run forward until it reaches the open surface at the right side. After the shock passage, the perturbation changes its phase, and the spike starts to grow from a hollow. However, for a shock wave with piston velocity $u_{p}=0.5$ we did not observe the spike growth after the hollow collapsed (see the dashed line approaching zero in Fig. 5). This indicates a threshold-type dynamics of the generation of the material flow around the perturbation. In contrast to the light-to-heavy system, in this case the growth-rate of the spike amplitude is slowing down to zero when the rarefaction wave from the open surface gets back to the piston. Multiple intersections of the transmitted non-planar shock waves (e.g. as seen in the second snapshot in Fig. 4) create local areas with an increased stress and temperature around the growing spike ("hot spots") where the material might become either molten or amorphous.

In modeling the shock-induced instability of the perturbed copper surface we generate a shock wave along the [110] direction. A characteristic perturbation in the largest-size simulation $\left(\sim 6 \times 10^{6}\right.$ atoms) has a sinusoidal-like shape with the

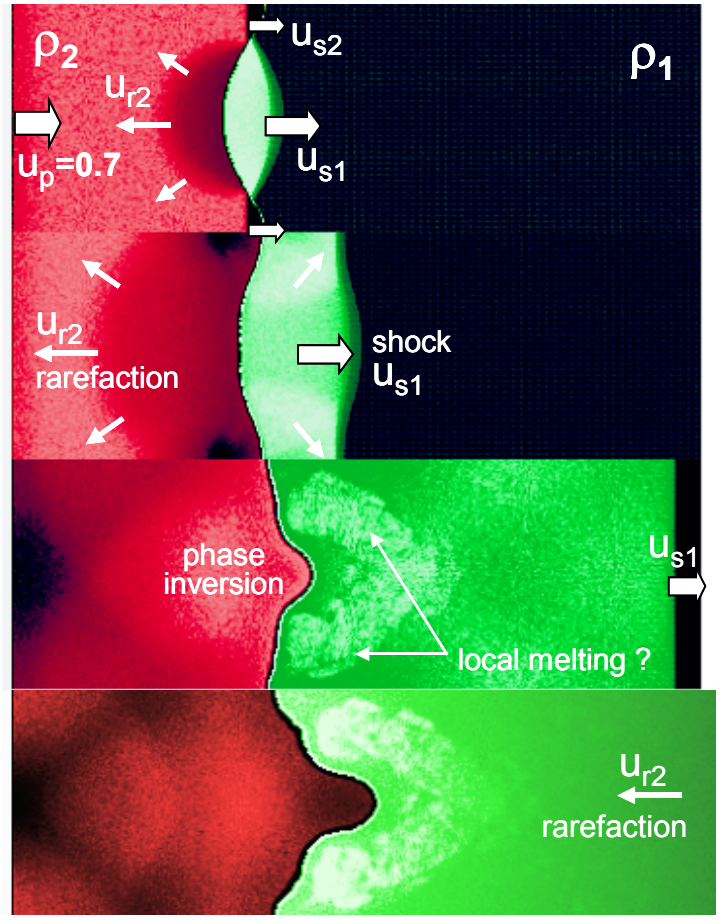

FIGURE 4. Snapshots from the simulation of phase change and growth of the perturbation during shock propagation in heavy-to-light solid at the piston velocity $u_{p}=0.7 \mathrm{mdu} ; \eta=\lambda / 3=36 \mathrm{~nm}$. The spike arising from the valley continues to grow until a rarefaction wave from the open right side comes down to the piston.

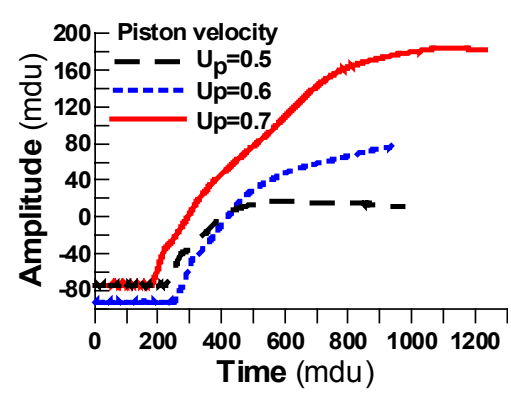

FIGURE 5. Evolution of the perturbation amplitude during heavy-to-light shock passage for three piston velocities: $0.5,0.6$, and $0.7 \mathrm{mdu} ; \eta=\lambda / 3=36 \mathrm{~nm}$. The amplitude sign change indicates a phase inversion of the perturbation

wavelength $\lambda=74 \mathrm{~nm}$ and the amplitude $\eta=\lambda / 4$. The piston velocity is chosen in a range of $1-2.8$ $\mathrm{km} / \mathrm{s}$ in order to keep it below the melting limit of 

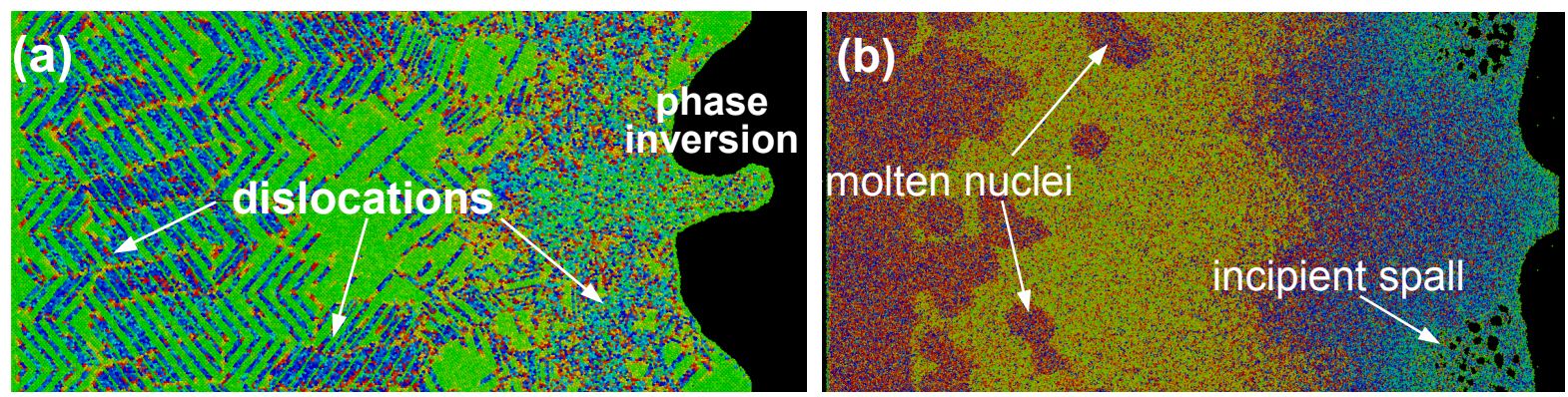

FIGURE 6. Two simulations of the RMI of the copper-vacuum interface with different perturbations parameters and piston velocities: (a) [100] shock, $\lambda=\eta=36 \mathrm{~nm}, \mathrm{u}_{\mathrm{p}}=1 \mathrm{~km} / \mathrm{s}, \mathrm{u}_{\mathrm{s}} \approx 5.5 \mathrm{~km} / \mathrm{s}, \mathrm{P}_{\mathrm{s}} \approx 50 \mathrm{GPa}$; (b) [110] shock, $\lambda=4 \eta=74 \mathrm{~nm}, \mathrm{u}_{\mathrm{p}}=2.8 \mathrm{~km} / \mathrm{s}, \mathrm{u}_{\mathrm{s}}$ $\approx 8.2 \mathrm{~km} / \mathrm{s}, \mathrm{P}_{\mathrm{s}} \approx 200 \mathrm{GPa}$. In both cases, the phase inversion has been observed as a growth of the spike from the hollow.

$\sim 3 \mathrm{~km} / \mathrm{s}$ for the shocked bulk copper [12]. Figure 6 shows two different examples of the instability growth when the shock wave reaches the open surface and the rarefaction wave moves backward into the bulk. In the first case (see Fig. 6a), we set the ratio $\lambda / \eta=1$, which corresponds to a high degree of non-linearity even at the very early stages of instability. As a result, we were able to see the spike growth at the velocity of the piston $u_{p}=1 \mathrm{~km} / \mathrm{s}$. Remarkably, the cup of the spike displays a high density of defects in comparison to dislocations in the bulk crystal, indicating possible amorphization of the material inside the spike and beneath which can facilitate the plastic flow around it and can sustain its growth.

In case of smaller perturbation amplitude $\eta=$ $\lambda / 4$ we observed the spike growth for two different piston velocities of 2.0 and $2.8 \mathrm{~km} / \mathrm{s}$. In the last case, with the bulk pressure close to the calculated shock melting limit of $220 \mathrm{GPa}$ [12], we noticed an appearance of local spots that might be nuclei of molten material. In addition, the void formation and nucleation with incipient spall have been detected underneath the hills of the initial perturbation during its phase inversion and spike growth at the unloading surface. Such dynamics is not observed in the simulations of shock unloading at a planar surface, and we relate it to the intersections of the non-planar rarefaction waves moving down from the perturbed interface.

In summary, we have performed the first MD simulations of the Richtmyer-Meshkov instability in solids for different types of the interface and have studied various scenarios of the perturbation growth as well as its dependence on the shock orientation and strength. Such simulations provides unmatched insight into the nanoscale structure of the shocked material, and can determine the mechanism governing the instability growth in solids as well as lead to understanding of the material properties at a very high strain rates.

\section{ACKNOWLEDGEMENTS}

SVZ is supported by DOE ASC, ONR and ARO MURI. Work at LLNL was performed under the auspices of the U.S. Department of Energy by University of California, Lawrence Livermore National Laboratory under contract of No.W-7405Eng-48, LDRD 04-ERD-021. SIA is supported by NRL and by DOE ASC, contract \#B523820.

\section{REFERENCES}

1. Barnes J.F., Blewett P.J., McQuenn R.G., Meyer K.A., and Venable D., J. Appl. Phys. 45, 727 (1974)

2. Barnes J.F., Janney D.H., London R.K., Meyer K.A., and Sharp D.H., J. Appl. Phys. 51, 4678 (1980).

3. Colvin J.D., Legrand M., Remington B.A., and Weber S.V., J. Appl. Phys. 93, 5287-5301 (2003).

4. Remington B.A. et al, Metal. Mater. Trans. A 35A, 2587-2607 (2004).

5. Plohr B.J., Sharp D.H. ZAMP, 49, 786 (1998).

6. Wouchuk J.G. and Nishihara K., Phys. Plasmas. 4, 1028 (1997).

7. Terrones G., Phys. Rev. E, 71, 036306 (2005).

8. Germann, T.C., Hammerberg, J.E., and Holian, B.L. in Shock Compression of Condensed Matter - 2003, edited by M. D. Furnish, Y. M. Gupta, and J. W. Forbes, AIP Conf. Proc. 706, 285-288 (2004).

9. Kadau K., et al, PNAS, 101 (16), 5851-5855 (2004).

10. Zhakhovskii V.V., Zybin S.V., Nishihara K., and Anisimov S.I., Phys. Rev. Lett. 83, 1175 (1999).

11. Mishin et al, Phys.Rev.B 63, 224106 (2003). 
12. Bringa E.M., Cazamias J.U., Erhart P., Stolken J., Tanushev N., Wirth B.D., Rudd R.E., and Caturla M.J., J. Appl. Phys. 96, 3793 (2004).

13. Erhart P., Bringa E.M., Kumar M., Albe K. Phys. Rev. B 72, 052104 (2005) 